\title{
Guiding shear bands in bulk metallic glasses using stress fields: A perspective from the activation of flow units
}

\author{
K. Kosiba $\odot,{ }^{1, *}$ S. Scudino $\odot,{ }^{1, \dagger}$ J. Bednarcik, ${ }^{2,3}$ J. Bian, ${ }^{4}$ G. Liu, ${ }^{4}$ U. Kühn, ${ }^{1}$ and S. Pauly ${ }^{1,5}$ \\ ${ }^{1}$ Leibniz. IFW Dresden, Institute for Complex Materials, Helmholtzstraße 20, 01069 Dresden, Germany \\ ${ }^{2}$ P. J. Safarik University in Košice, Institute of Physics, Department of Condensed Matter Physics, Park Angelinum 9, 04154 Košice, Slovakia \\ ${ }^{3}$ Slovak Academy of Science, Institute of Experimental Physics, Watsonova 47, 040 01, Košice, Slovakia \\ ${ }^{4}$ State Key Laboratory for Mechanical Behaviors of Materials, School of Materials Science and Engineering, Xi'an Jiaotong University, \\ West Xianning Road, Xi'an, 710049 Shaanxi, People's Republic of China \\ ${ }^{5}$ University of Applied Sciences Aschaffenburg, Würzburger Straße 45, 63743 Aschaffenburg, Germany
}

(Received 19 June 2020; accepted 24 September 2020; published 19 October 2020)

\begin{abstract}
Controlling shear band propagation is the key to obtain ductile metallic glasses. Here, we use a residual stress field to vary the direction of shear band propagation. We ascribe this behavior to the effect of the stress field on the activation of shear transformation zones (STZs) along their characteristic direction and we quantify this contribution to the energy of the process. Because of the progressively adverse orientation of the stress field, the energy stored as shear in the STZ decreases to a level where shear band propagation at alternative angles becomes energetically more favorable.
\end{abstract}

DOI: 10.1103/PhysRevB.102.134113

\section{INTRODUCTION}

Plastic deformation of bulk metallic glasses (BMGs) at room temperature occurs through the generation of highly localized shear bands propagating at the microscale in an approximately straight manner [1,2] (although shear band deflections have been observed at the nanoscale $[3,4])$. Recent molecular-dynamics simulations [5] indicate that this behavior results from the sequential activation of shear transformation zones (STZs, the elementary units of plasticity in BMGs $[6,7])$ along a specific direction. This mechanism is schematically shown in Fig. 1(a), where STZs are connected by springs. According to this model, the stress field generated by an activated STZ in the surrounding material [represented by the deformed springs in Fig. 1(a)] is the medium that progressively triggers the activation of the adjacent potential STZ $[5,8]$. The reiteration of this step, as in the domino effect, allows a plastic flow unit to be transported across the sample $[5,9]$.

For (ideally) homogeneous BMGs, where no structural heterogeneities, such as local variations of density [9-11] and atomic cluster topology [12] or presence of residual stresses [13], disturb this sequential flow process, the activation of an STZ can be represented by a structural distortion with a characteristic orientation $\left[\sigma^{S T Z}\right.$ in Fig. 1(b)]. In this case, the

\footnotetext{
*k.kosiba@ifw-dresden.de

†s.scudino@ifw-dresden.de
}

Published by the American Physical Society under the terms of the Creative Commons Attribution 4.0 International license. Further distribution of this work must maintain attribution to the author(s) and the published article's title, journal citation, and DOI. distortion of each STZ is identical along the nascent shear band, determining the relatively straight trajectory of the band while it spans the entire specimen [1]. If this is true, then it would be possible to control the shear band path and the overall plastic deformation of BMGs by manipulating the orientation of STZ activation. This hypothesis can be tested experimentally by introducing structural perturbations along the path of a shear band.

Here, we explore this strategy by generating a smoothly varying residual elastic stress field in a $\mathrm{Cu}_{44} \mathrm{Zr}_{44} \mathrm{Al}_{8} \mathrm{Hf}_{2} \mathrm{Co}_{2}$ BMG using flash annealing. Flash annealing represents a unique tool for introducing elastic stress fields into BMGs while avoiding the formation of shear bands [9] as, in contrast, occurs for shot-peened, cold-rolled, or imprinted BMGs [13-17]. This permits us to use the trajectory of shear bands as a local probe in order to study the influence of stress fields on the underlying STZ activation mechanism without the interference resulting from the stress fields generated by preexisting bands [8]. Our approach is schematically shown in Fig. 1(c), where a residual stress field $\left(\sigma^{F A}\right)$ is used to change the structural distortion that results from the local shear transformation event characteristic of the homogeneous BMG.

\section{EXPERIMENT}

Elastic stress fields were generated in $\mathrm{Cu}_{44} \mathrm{Zr}_{44} \mathrm{Al}_{8} \mathrm{Hf}_{2} \mathrm{Co}_{2}$ (at. \%) amorphous cylindrical specimens by flash annealing. This composition was selected for our investigation because it shows good glass-forming ability and can be synthesized in a reproducible manner. Ingots with a mass of around $20 \mathrm{~g}$ having this composition were prepared by arc melting using high-purity elements (more than $99.5 \%$ purity). The prealloys were repeatedly arc melted under a Ti-gettered Ar atmosphere 
(a)

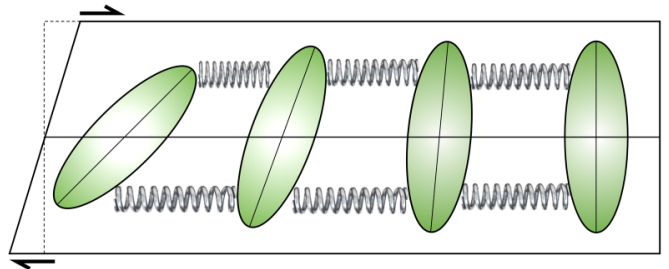

(b) Homogeneous

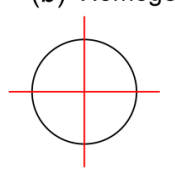

$$
\underset{\text { STZ activation }}{\stackrel{\sigma^{S T Z}}{\longrightarrow}}
$$

(c) Heterogeneous
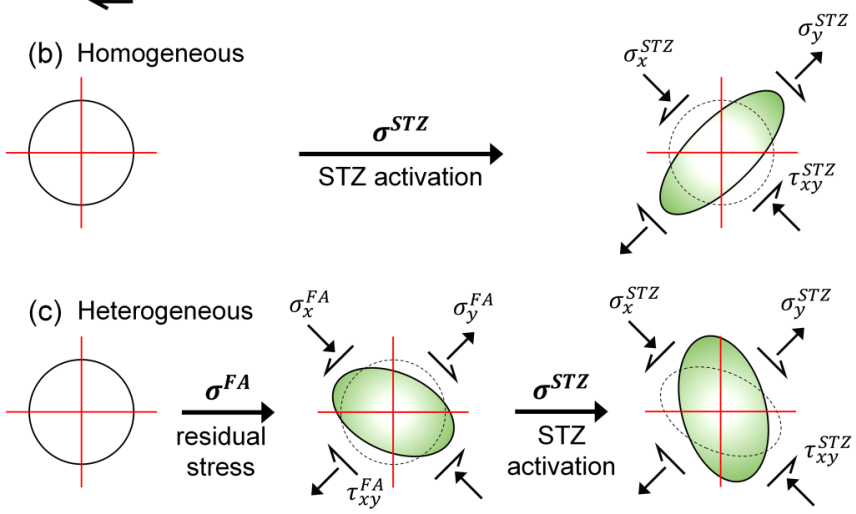

FIG. 1. (a) Schematic representation of the dominolike STZ activation mechanism. (b) Activation of an STZ in a homogeneous BMG represented by the application of the stress tensor $\boldsymbol{\sigma}^{S T Z}$ to an unstressed potential STZ (circles). (c) Effect of a superimposing residual stress field $\left(\sigma^{F A}\right)$ on the directionality of the structural distortion given by $\sigma^{S T Z}$. Distorted STZs are represented by ellipses.

to ensure chemical homogeneity. BMG cylinders with a diameter of $3 \mathrm{~mm}$ were synthesized by suction casting the arc melted ingot in a copper mold.

Flash annealing was carried out in a custom-made device developed in house [18]. The device heats electrically conductive materials via electromagnetic induction at defined rates (up to $200 \mathrm{~K} / \mathrm{s}$ for bulk metallic glasses) to a given target ejection temperature. Because of kinetic constraints, the crystallization temperature of a glass can be shifted to higher values with increasing the heating rate [19-21]. High heating rates are thus essential to achieve high temperatures avoiding crystallization of the glass. Subsequent quenching in a water bath at a rate exceeding $1000 \mathrm{~K} / \mathrm{s}$ preserves the structural state characteristic of the ejection temperature. During heating, the samples are flushed using an Ar shower to minimize surface oxidation at high temperatures. In the present work, the flash-annealing procedure consisted of heating the $\mathrm{Cu}_{44} \mathrm{Zr}_{44} \mathrm{Al}_{8} \mathrm{Hf}_{2} \mathrm{Co}_{2}$ specimens at about $63 \mathrm{~K} / \mathrm{s}$ via electromagnetic induction to $861 \mathrm{~K}$, between the glass transition and crystallization temperatures where the material is a supercooled liquid. This ejection temperature is about $60 \mathrm{~K}$ higher than the crystallization temperature observed at conventional heating rates used in calorimetry measurements [18]. Subsequent quenching in water allows the material to revitrify (see Supplemental Material, Fig. S1 [22]).

High-energy x-ray diffraction (HEXRD) experiments of as-cast and flash-annealed $\mathrm{Cu}_{44} \mathrm{Zr}_{44} \mathrm{Al}_{8} \mathrm{Hf}_{2} \mathrm{Co}_{2}$ glassy samples were performed in transmission using a highenergy monochromatic synchrotron beam (wavelength $\lambda=$ $0.2066 \AA$; beam size $25 \times 25 \mu \mathrm{m}^{2}$ ) at the P02.1 beamline at the Deutsches Elektronen-Synchrotron (DESY). Prior to characterization, the cylindrical specimens were ground down
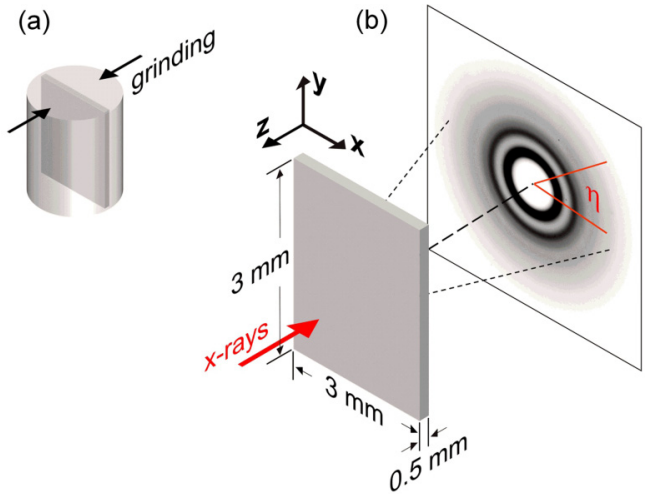

FIG. 2. Schematic of the HEXRD setup, sample dimensions, and coordinate system used in the experiment.

to foils with area of $3 \times 3 \mathrm{~mm}^{2}$ and uniform thickness of $0.5 \mathrm{~mm}$ [Fig. 2(a)].

2D diffraction patterns were recorded every $100 \mu \mathrm{m}$ along the $x$ and $y$ axes covering an area of about $3 \times 3 \mathrm{~mm}^{2}$ (acquisition time $10 \mathrm{~s}$ ) using a Perkin Elmer 1621 area detector. The patterns were integrated in $10^{\circ}$ azimuthal slices between 0 and $360^{\circ}$ using the PYFAI software package [23] to give intensity distributions $I(q)$ as a function of the scattering vector $q$ for each $(x, y)$ position and azimuthal integration angle $\eta$ [Fig. 2(b)]. In order to optimize the diffraction experiment, we focused our investigation on the variation of the first scattering maximum $\left(q_{1}\right)$ in reciprocal space. The $q_{1}$ peak can be measured more quickly using a relatively longer sample to detector distance that reduces systematic errors and can be reliably used to examine the structural changes taking place in the medium-range order of glassy metals [24]. The position of $q_{1}$ was evaluated by fitting using a pseudo-Voigt function. The strain can then be obtained through the shift of $q_{1}$ with respect to a reference value $q_{1}^{0}$ as

$$
\varepsilon=\frac{\left[q_{1}^{0}-q_{1}(\eta, x, y)\right]}{q_{1}(\eta, x, y)},
$$

which depends on the azimuthal angle $\eta$ and on the $(x, y)$ position. The flash-annealed BMG shows stronger variations of $q_{1}$ compared with the as-cast sample (see Supplemental Material, Fig. S2 [22]). We therefore selected $q_{1}^{0}$ as the average peak position of the as-cast BMG (averaged over 732 points).

The components of the strain tensor $\boldsymbol{\varepsilon}^{\boldsymbol{F A}}\left(\varepsilon_{x}^{F A}, \varepsilon_{y}^{F A}\right.$ and $\left.\varepsilon_{x y}^{F A}\right)$ were determined for each point $(x, y)$ according to the method described in Poulsen et al. [25] by fitting the angular variation of $\varepsilon$ to the expression

$$
\begin{aligned}
\varepsilon & =\varepsilon_{x}^{F A} \cos ^{2} \eta-2 \varepsilon_{x y}^{F A} \sin \eta \cos \eta+\varepsilon_{y}^{F A} \sin ^{2} \eta \\
\text { with } \eta & =10,20 \ldots 360^{\circ}
\end{aligned}
$$

which assumes zero strain along the x-ray beam direction (thin direction on the sample). The stress tensor $\sigma^{F A}$ was determined from the generalized Hooke's law $\sigma^{\boldsymbol{F A}}=\boldsymbol{C} \cdot \boldsymbol{\varepsilon}^{\boldsymbol{F A}}$, where $\boldsymbol{C}$ is the stiffness tensor. For the present case, this expression 
can be written in matrix form as

$$
\begin{aligned}
\left(\begin{array}{l}
\sigma_{x}^{F A} \\
\sigma_{y}^{F A} \\
\tau_{x y}^{F A}
\end{array}\right)= & \frac{E}{(1+v)(1-2 v)}\left(\begin{array}{ccc}
1-v & v & 0 \\
v & 1-v & 0 \\
0 & 0 & \frac{(1-2 v)}{2}
\end{array}\right) \\
& \times\left(\begin{array}{c}
\varepsilon_{x}^{F A} \\
\varepsilon_{y}^{F A} \\
2 \varepsilon_{x y}^{F A}
\end{array}\right)
\end{aligned}
$$

where $E=90.5 \pm 0.3 \mathrm{GPa}$ and $v=0.371 \pm 0.00$ are Young's modulus and Poisson's ratio of the flashannealed BMG obtained experimentally by ultrasonic measurements.

The eigenvalues of the stress tensor $\sigma^{F A}(\lambda)$, which correspond to the magnitudes of the principal stresses, were calculated by solving the characteristic equation, $\left|\sigma^{F A}-\lambda I\right|=0$, where $I$ is the identity matrix. The eigenvectors $v$, which give the directions of the principal stress axes, were then calculated by solving the equations $\left|\sigma^{F A}-\lambda I\right| v=0$.

The generation of elastic stress fields by flash annealing was also investigated by complementary simulations via finite-element method (FEM) using the software COMSOL MULTIPHYSICS. The FEM simulations have been designed to mimic the flash-annealing process as a sequence of rapid heating $(63 \mathrm{~K} / \mathrm{s})$ to the target ejection temperature $(861 \mathrm{~K})$ via electromagnetic induction followed by immediate water quenching (cooling rate $1000 \mathrm{~K} / \mathrm{s}$ ) of $\mathrm{Cu}_{44} \mathrm{Zr}_{44} \mathrm{Al}_{8} \mathrm{Hf}_{2} \mathrm{Co}_{2}$ cylindrical specimens with dimensions of $3 \mathrm{~mm}$ diameter and $6 \mathrm{~mm}$ length. Additional details about FEM simulation and the necessary input data are given in Supplemental Material, Figs. S3-S6 [22].

The shear band morphology after compression tests was analyzed by inspecting semicylindrical specimens via scanning electron microscopy (SEM) using an SEM-FEG Gemini 1530 microscope. Thereby, we followed an approach used in previous works (see Supplemental Material, Fig. S8) [26,27].

\section{RESULTS AND DISCUSSION}

The position-resolved stress tensor $\boldsymbol{\sigma}^{F A}\left(\sigma_{x}^{F A}, \tau_{x y}^{F A}\right.$, and $\sigma_{y}^{F A}$ ) for the flash-annealed BMG derived from HEXRD is shown in Fig. 3(a). The components $\sigma_{x}^{F A}$ and $\sigma_{y}^{F A}$ exhibit a Y-shaped distribution. The stress is compressive (or slightly tensile) in the vicinity of the sample edges and decreases towards the center, where it becomes increasingly more tensile. The shear component $\tau_{x y}^{F A}$ is largest near the sample corners and decreases moving down along the $y$ axis. Although the magnitude of the stresses obtained from the FEM simulations [Fig. 3(b)] is overall lower compared to the HEXRD results, the stress fields are very similar, supporting that the experimental data in Fig. 3(a) represent well the stresses generated by flash annealing.

Room-temperature compression tests reveal that the flashannealed specimens yield at lower stress, subsequently work harden, and show a four times larger plastic strain than the as-cast BMG (see Supplemental Material, Fig. S7). Although more effective methods for enhancing plasticity of BMGs are known [28-36], flash annealing has the merit to create stress fields without sacrificing structural integrity by forming shear bands, which would be the preferential location for plastic deformation during subsequent mechanical loading [37,38]. Also, flash annealing is very reliable in creating predictable stress fields [Fig. 3(b)], which then leads to reproducible mechanical properties and related shear band morphology (see Supplemental Material, Table S2). Finally, this method opens the path to the design of stress fields by properly varying the characteristics of the magnetic field and the cooling conditions via either changing the working coil geometry or the shape of the BMG specimens. As-cast and flash-annealed BMGs are fully amorphous (see Supplemental Material, Fig. S1), so that deformation is based on shear band formation for both. Yet, shear banding must occur in a different manner and we will dwell on it next.

Figures 3(c) and 3(d) show the shear band morphology for the as-cast and flash-annealed semicylindrical specimens after compression. Only a few shear bands with an angle of $\theta=42^{\circ}$ with respect to the $x$ axis form in the as-cast BMG [Fig. 3(c)], in agreement with the shear band angle of about $45^{\circ}$ typically observed in BMGs under compressive loading [1]. The flash-annealed BMG shows a higher density of shear bands [Fig. 3(d) and Supplemental Material, Fig. S8]. These bands initially propagate at $42^{\circ}$ at the borders of the sample and then progressively change their direction forming an angle of $\theta=0^{\circ}$ at about $100 \mu \mathrm{m}$ away from the edge. Shear banding at $\theta=0^{\circ}$ is not due to the semicylindrical geometry of the specimens as they are formed in the cylindrical samples as well (see Supplemental Material, Fig. S9).

Shear band orientation in the flash-annealed BMG conforms to the eigenvectors of the stress tensor, which give the magnitude and direction of the principal stresses [compare Figs. 3(d) and 3(e)], indicating that stress fields can be used to effectively guide shear bands. This behavior could be rationalized in terms of fictive temperature $T_{f}$, which correlates the properties of a glass at room temperature (e.g., plastic deformation or free volume) with the properties of the supercooled liquid via the cooling rate $[39,40]$. In the flashannealed material, the cooling rate (and therefore the fictive temperature) varies along the radius of the specimen, implying that different regions of the sample are more or less ductile depending on the local value of $T_{f}$. Although this interpretation is suggestive and useful to describe the overall properties of BMG samples [39,40], a scalar and thus nondirectional quantity like $T_{f}$ (or the corresponding free-volume content) is unable to accurately explain directional phenomena, as the shear band morphology shown in Fig. 2(d). A better approach to understand this aspect is to consider the change of shear band direction in the flash-annealed glass as the microscopic manifestation of a change in STZ activation at the nanoscale. Assuming a sequential STZ activation mechanism based on the dominolike effect sketched in Fig. 1(a), the contribution of the residual stress makes toppling of STZs along the characteristic direction of the as-cast BMG more difficult. This aspect can be better understood by considering the contribution of a superimposing stress field to the energetics of STZ activation. To do so, we start from the cooperative shear model (CSM) [41], which quantifies the energy barrier for STZ activation in homogeneous metallic glasses (no residual 

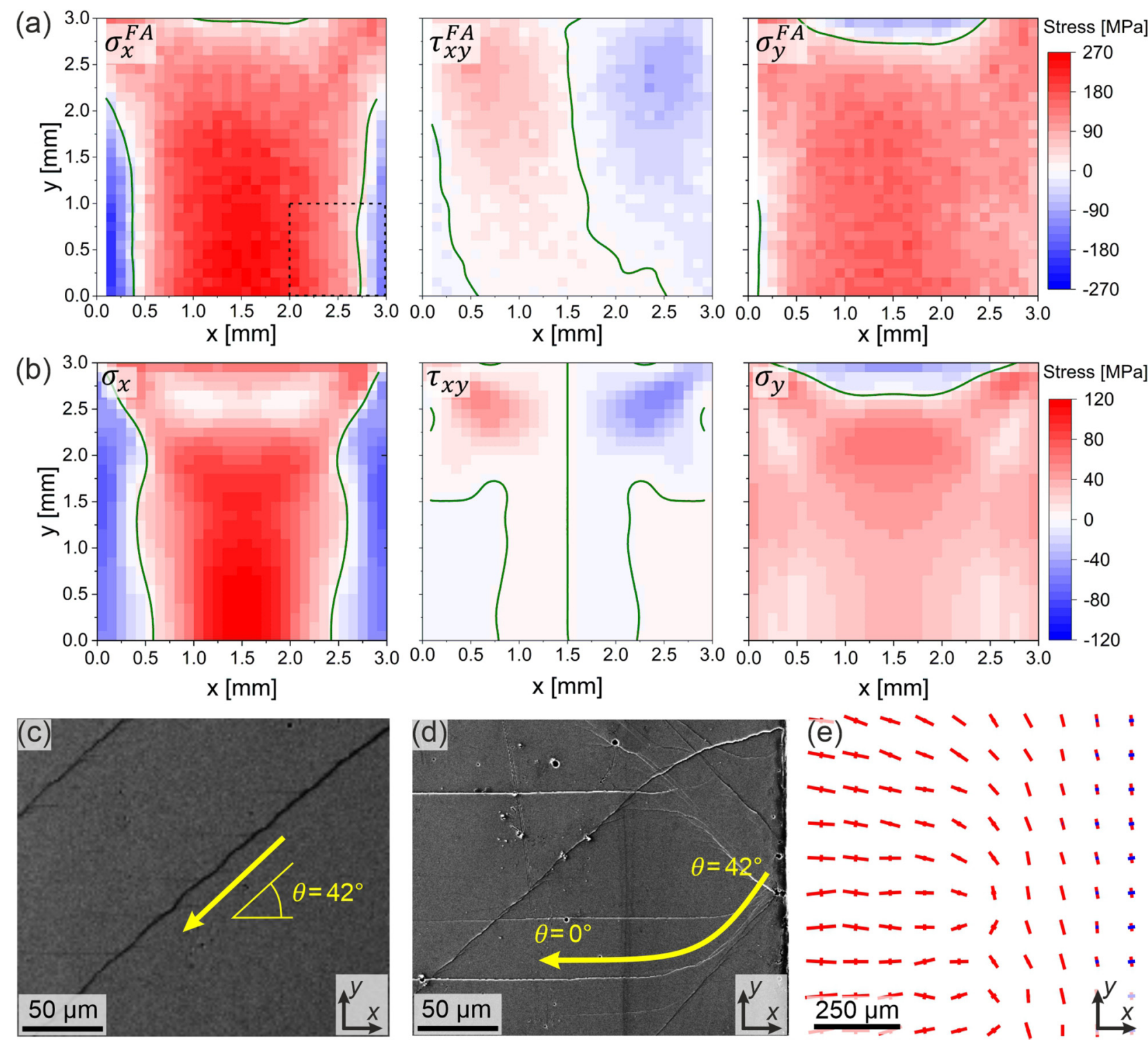

FIG. 3. Position-resolved stress tensors for the flash-annealed $\mathrm{Cu}_{44} \mathrm{Zr}_{44} \mathrm{Al}_{8} \mathrm{Hf}_{2} \mathrm{Co}_{2} \mathrm{BMG}$ obtained by (a) HEXRD and (b) FEM. Shear band morphology at early stages of plastic deformation for (c) as-cast and (d) flash-annealed semicylindrical specimens. Shear band propagation at $42^{\circ}$, characteristic of the as-cast material, changes in the flash-annealed BMG [indicated by the arrow in (d)]. (e) Stress eigenvectors derived from HEXRD, showing the correlation between principal stresses and shear band direction; compressive stresses are blue and tensile stresses are red; the length of the eigenvectors gives the magnitude of the principal stresses. The area covered in (e) corresponds to the region delimited by the dashed black box in (a).

stresses) as

$$
\begin{aligned}
W^{\mathrm{CSM}}= & \varphi \zeta \Omega=\left(\frac{8}{\pi^{2}}\right)\left[G\left(\frac{\tau_{c}}{2 G}\right)^{2}\right] \zeta \Omega=\left(\frac{8}{\pi^{2}}\right) \\
& \times\left[G\left(\frac{\sigma_{A} \sin \theta \cos \theta}{2 G}\right)^{2}\right] \zeta \Omega,
\end{aligned}
$$

where $\varphi$ is the total barrier energy density, $G$ is the shear modulus, $\Omega$ is the STZ volume, $\zeta=3<$ is a correction factor, $\tau_{c}$ is the critical shear stress resolved along the direction given by the angle $\theta$, and $\sigma_{A}$ is the macroscopic stress at yield acting along the loading $y$ axis. The CSM only considers the uniaxially applied stress $\sigma_{A}$. In order to account for a superimposing stress field in which the other components are nonzero, such as the field in Fig. 3(a), we extend the CSM to include the interaction of the stress field with all stress components characterizing STZ activation. The model can then be generalized to consider the variation of the tensorial shear stress with $\theta$ to predict the shear band angle.

In the following, we explore this aspect by first selecting a reference stress tensor $\left(\boldsymbol{\sigma}^{S T Z}\right)$ representing the stress field characteristic of a shear transformation in a homogeneous BMG. We can then quantify the superimposition of $\boldsymbol{\sigma}^{S T Z}$ with the stress field generated locally by flash annealing $\left[\sigma^{F A}\right.$, Fig. 3(a)] using

$$
\begin{aligned}
\boldsymbol{\sigma}^{e f} & =\left(\begin{array}{cc}
\sigma_{x}^{e f} & \tau_{x y}^{e f} \\
\tau_{x y}^{e f} & \sigma_{y}^{e f}
\end{array}\right)=\sigma^{\boldsymbol{F A}}+\boldsymbol{\sigma}^{S T Z} \\
& =\left(\begin{array}{cc}
\left(\sigma_{x}^{F A}+\sigma_{x}^{S T Z}\right) & \left(\tau_{x y}^{F A}+\tau_{x y}^{S T Z}\right) \\
\left(\tau_{x y}^{F A}+\tau_{x y}^{S T Z}\right) & \left(\sigma_{y}^{F A}+\sigma_{y}^{S T Z}\right)
\end{array}\right),
\end{aligned}
$$



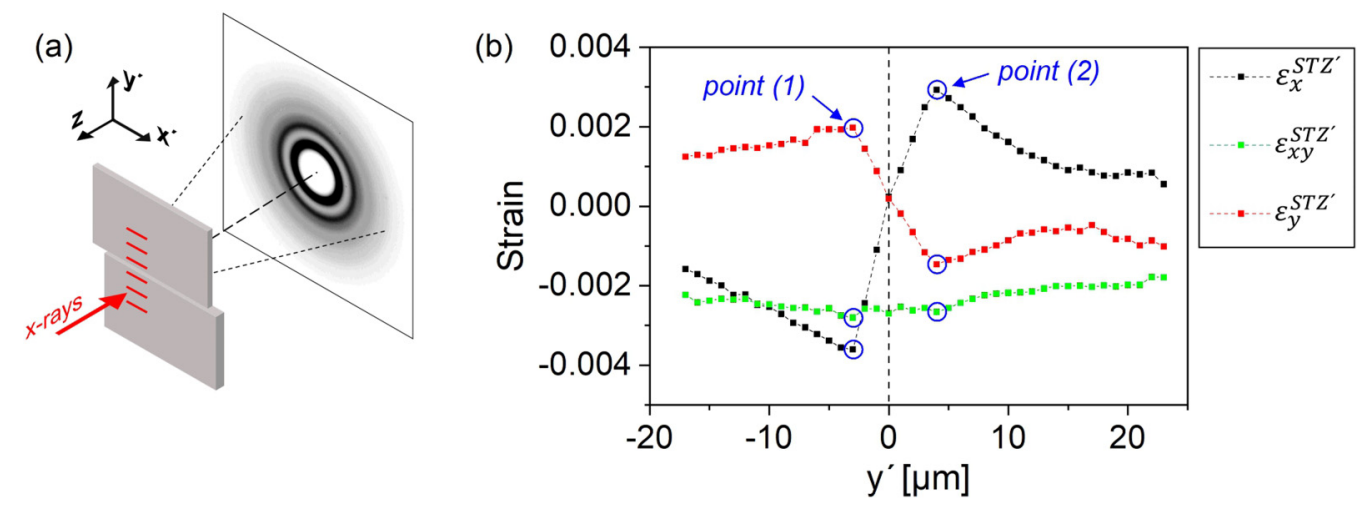

FIG. 4. (a) Experimental HEXRD setup and coordinate system. (b) Strain variation across a shear band determined by HEXRD. The vertical dashed line marks the position of the shear band.

where the stress tensor $\sigma^{\text {ef }}$ represents the effective stress acting on a potential STZ at a given position in the glass. This approach is equivalent to the combination of the structural distortions schematically shown in Fig. 1(c). The shear component of $\sigma^{e f}$, which is the effective shear stress an untransformed STZ "feels," can be expressed as a function of $\theta$ (the angle between the shear band and the $x$ axis) as [42]

$$
\tau_{x y}^{\prime e f}=-\frac{\sigma_{x}^{e f}-\sigma_{y}^{e f}}{2} \sin 2 \theta+\tau_{x y}{ }^{e f} \cos 2 \theta,
$$

which is then substituted in Eq. (4) to give

$$
\begin{aligned}
W^{e f} & =W^{S T Z}+W^{F A} \\
& =\left(\frac{8}{\pi^{2}}\right)\left[G\left(\frac{-\frac{\sigma_{x}^{e f}-\sigma_{y}^{e f}}{2} \sin 2 \theta+\tau_{x y}^{e f} \cos 2 \theta}{2 G}\right)^{2}\right] \zeta \Omega .
\end{aligned}
$$

Equation (7) should be interpreted as the elastic energy stored as shear within an STZ volume rather than the energy barrier for STZ activation predicted by Eq. (4). For the CSM both views are equivalent because, on loading, the homogeneous glass always stores the critical shear energy at $\theta=45^{\circ}$, where shear bands typically form. Additionally, in a homogeneous BMG each STZ experiences the same applied stress, meaning that at the macroscopic yield $\sigma_{A}$ the critical energy for STZ activation given by Eq. (4) can be nominally reached everywhere in the specimen. The scenario is different in a heterogeneous BMG, where the stress acting on a potential STZ (and thus the corresponding critical energy stored as shear) varies locally. We use $\sigma^{S T Z}$ as a local probe to identify the regions where the critical shear energy is reached. This energy naturally depends on the angle $\theta$ but also on the position within the sample due to the nonuniform residual stress field [Fig. 3(a)], meaning that shear bands might propagate at angles deviating from the $45^{\circ}$ typical of homogeneous BMGs.

The difficulty now is to experimentally determine $\sigma^{S T Z}$. The selection of the stress tensor representing the activation of an STZ in a homogeneous BMG $\left(\sigma^{S T Z}\right)$ is a critical aspect of this work, as it sets the reference state for evaluating the effect of a superimposing stress field. To be characteristic of STZ activation, $\sigma^{S T Z}$ should represent the stress state within a shear band. Unfortunately, because of technical limitations, no experimental quantification of the stress tensor within a shear band has been possible until now. The structural distortion resulting from STZ activation within a shear band, however, leaves traces in the surrounding elastic matrix observable by XRD experiments and molecular-dynamics simulations [8]. We use this structural signature to estimate $\sigma^{S T Z}$.

The evaluation of the strain across a shear band by HEXRD (Fig. 4(a); for additional details see Refs. [8]) revealed that shear band formation generates a characteristic sigmoidal strain profile in the surrounding material perceptible at a distance of several microns from the band [Fig. 4(b)]. This strain profile is the mark left by STZ activation within a shear band that can be also observed by molecular-dynamics simulations $[5,8]$.

Because the strain decreases in the vicinity of the shear band (most likely due to heat release and relaxation near the band), in our attempt to estimate $\sigma^{S T Z}$ we select the strain tensor $\boldsymbol{\varepsilon}^{S T Z^{\prime}}$ corresponding to point (1) in Fig. 4(b), which is expected to better represent the structural distortion occurring within the shear band [similar results can be obtained by selecting point (2)]

$$
\varepsilon^{S T Z^{\prime}}=\left(\begin{array}{ll}
\varepsilon_{x}^{S T Z^{\prime}} & \varepsilon_{x y}^{S T Z^{\prime}} \\
\varepsilon_{x y}^{S T Z^{\prime}} & \varepsilon_{y}^{S T Z^{\prime}}
\end{array}\right)=\left(\begin{array}{cc}
-0.0036 & -0.0028 \\
-0.0028 & 0.00198
\end{array}\right) .
$$

In the experiment leading to Fig. 4(b) $[5,8]$, the shear band was parallel to the $x^{\prime}$ axis. The strain tensor $\varepsilon^{S T Z^{\prime}}$, therefore, has to be rotated by $45^{\circ}$ to be compatible with the $x-y$ coordinate system, where shear bands propagate at $45^{\circ}$ [Figs. 2 and $3(\mathrm{c})]$, as

$$
\begin{aligned}
\boldsymbol{\varepsilon}^{S T Z}= & \boldsymbol{R} \cdot \boldsymbol{\varepsilon}^{S T Z^{\prime}} \cdot \boldsymbol{R}^{T}=\left(\begin{array}{cc}
\cos \theta & \sin \theta \\
-\sin \theta & \cos \theta
\end{array}\right)\left(\begin{array}{ll}
\varepsilon_{x}^{S T Z^{\prime}} & \varepsilon_{x y}^{S T Z^{\prime}} \\
\varepsilon_{x y}^{S T Z^{\prime}} & \varepsilon_{y}^{S T Z^{\prime}}
\end{array}\right) \\
& \times\left(\begin{array}{cc}
\cos \theta & -\sin \theta \\
\sin \theta & \cos \theta
\end{array}\right) .
\end{aligned}
$$

where $\boldsymbol{R}$ is the rotation matrix and $\boldsymbol{R}^{\boldsymbol{T}}$ is its transpose. The strain components of $\varepsilon^{S T Z}$ in the $x$-y coordinate system as a function of $\varepsilon^{S T Z^{\prime}}$ and $\theta$, the angle between the $x-y$ and $x^{\prime}-y^{\prime}$ 

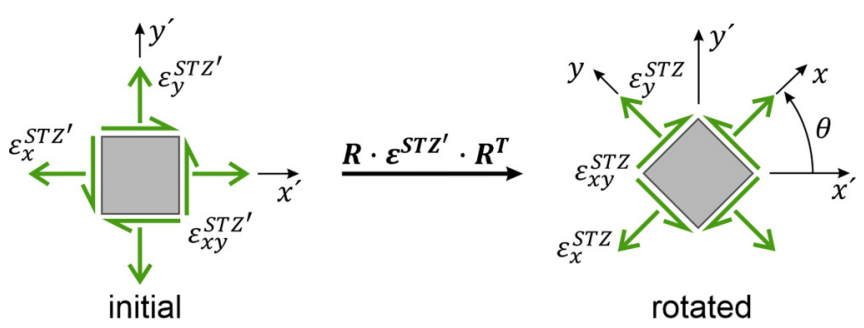

FIG. 5. Schematic illustrating the strain components of $\varepsilon^{S T Z^{\prime}}$ and $\boldsymbol{\varepsilon}^{S T Z}$ in the $x^{\prime}-y^{\prime}$ and $x-y$ coordinate systems. $\theta$ is the angle between the $x^{\prime}-y^{\prime}$ and $x-y$ frames.

frames (Fig. 5), is then given by [42]

$$
\begin{aligned}
& \varepsilon_{x}^{S T Z}=\frac{\varepsilon_{x}^{S T Z^{\prime}}+\varepsilon_{y}^{S T Z^{\prime}}}{2}+\frac{\varepsilon_{x}^{S T Z^{\prime}}-\varepsilon_{y}^{S T Z^{\prime}}}{2} \cos 2 \theta+\varepsilon_{x y}^{S T Z^{\prime}} \sin 2 \theta \\
& \varepsilon_{y}^{S T Z}=\frac{\varepsilon_{x}^{S T Z^{\prime}}+\varepsilon_{y}^{S T Z^{\prime}}}{2}-\frac{\varepsilon_{x}^{S T Z^{\prime}}-\varepsilon_{y}^{S T Z^{\prime}}}{2} \cos 2 \theta-\varepsilon_{x y}^{S T Z^{\prime}} \sin 2 \theta \\
& \varepsilon_{x y}^{S T Z}=-\frac{\varepsilon_{x}^{S T Z^{\prime}}-\varepsilon_{y}^{S T Z^{\prime}}}{2} \sin 2 \theta+\varepsilon_{x y}^{S T Z^{\prime}} \cos 2 \theta
\end{aligned}
$$

For $\theta=45^{\circ}$, the tensor $\varepsilon^{S T Z}$ in the $x-y$ coordinate system becomes

$$
\boldsymbol{\varepsilon}^{S T Z}=\left(\begin{array}{cc}
\varepsilon_{x}^{S T Z} & \varepsilon_{x y}^{S T Z} \\
\varepsilon_{x y}^{S T Z} & \varepsilon_{y}^{S T Z}
\end{array}\right)=\left(\begin{array}{cc}
-0.00361 & 0.00279 \\
0.00279 & 0.00199
\end{array}\right) .
$$

Finally, the corresponding stress tensor $\boldsymbol{\sigma}^{S T Z}$ in the $x-y$ coordinate system was calculated using Eq. (3), with $E=$ $92.4 \pm 0.2 \mathrm{GPa}$ and $v=0.368 \pm 0.001$ Young's modulus and Poisson's ratio of the as-cast BMG obtained experimentally by ultrasonic measurements, as

$$
\boldsymbol{\sigma}^{S T Z}=\left(\begin{array}{cc}
\sigma_{x}^{S T Z} & \tau_{x y}^{S T Z} \\
\tau_{x y}^{S T Z} & \sigma_{y}^{S T Z}
\end{array}\right)=\left(\begin{array}{cc}
-396 & 189 \\
189 & -18
\end{array}\right),
$$

where the stress is given in MPa.

From a plethora of experiments it is known that shear bands form under an angle of around $45^{\circ}$ (here: $42^{\circ}$ ) in structurally uniform metallic glasses $\left(\sigma^{F A}=0\right.$ or $\left.\sigma^{e f}=\sigma^{S T Z}\right)$. No structural features perturb the directionality of STZ activation given by $\boldsymbol{\sigma}^{S T Z}$ and shear bands propagate in a straight manner under compression [Fig. 3(c)]. In this case, the critical energy stored as shear given by Eq. (7) is a unique value characteristic of $W^{e f}=W^{S T Z}$ for $\theta=42^{\circ}$ that can be attained within the entire sample, as in the CSM. Additionally, for $\boldsymbol{\sigma}^{\boldsymbol{F A}}=0$, Eq. (7) can provide the critical energy for shear banding at $\theta=0^{\circ}$ or $90^{\circ}$. In the presence of a residual stress field $\left(\sigma^{F A} \neq 0\right)$, a potential STZ experiences an effective stress $\sigma^{e f}=\sigma^{S T Z}+\sigma^{F A}$. Depending on both the magnitude and direction of the field, an STZ volume might store lower energies than the critical energy required for shear banding at $42^{\circ}$. Equation (7) provides a tool to identify directions where higher energies are stored and, thus, to obtain energetically more favorable angles for shear band propagation.

Figure 6(a) shows the position-resolved energy stored as shear in the flash-annealed BMG derived using Eq. (7) for $\theta=42^{\circ}$ (where $\boldsymbol{\sigma}^{\boldsymbol{F A}}$ is the stress field in Fig. 2(a), $G=$ $E / 2(1+v)=33.8 \mathrm{GPa}$ is the shear modulus of the as-cast BMG derived from the ultrasound experimental values of $E=$ $92.4 \pm 0.2 \mathrm{GPa}$ and $v=0.368 \pm 0.001$, and $\Omega=3.85 \mathrm{~nm}^{3}$ is the STZ volume determined using the correlation between $v$ and $\Omega$ reported in Liu et al. [43]). At the edges of the specimen, where the direction of the superimposing stress field $\sigma^{F A}$
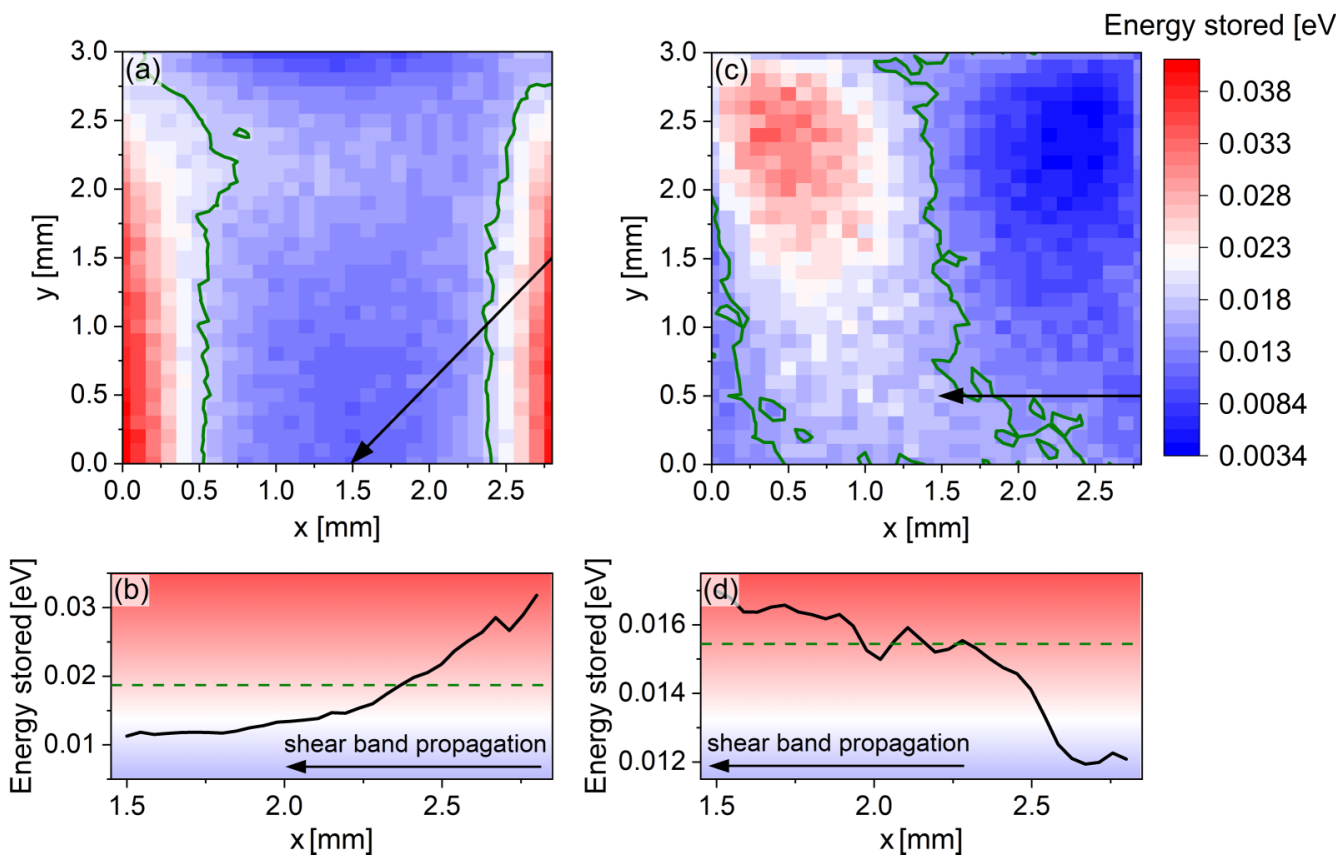

FIG. 6. Position-resolved effective energy stored as shear in the flash-annealed BMG derived using Eq. (7) for (a) $\theta=42^{\circ}$ and (c) $\theta=0^{\circ}$. (b), (d) Variation of effective energy for a shear band propagating from right to left [represented by the black arrows in (a) and (c), respectively]. The green lines in (a) and (b) mark the contour where $W^{e f}=W^{S T Z}(\theta)$, corresponding to the critical energy to be stored for STZ activation in the homogeneous material for $\theta=42^{\circ}$ and $0^{\circ}$, respectively. 
is favorably aligned with $\sigma^{S T Z}$, shear bands propagate at $42^{\circ}$ [Fig. 3(d)]. Here, potential STZs store higher energies than the critical value for shear banding at $42^{\circ}\left[W^{e f}=W^{S T Z}\left(42^{\circ}\right)=\right.$ $0.0187 \mathrm{eV}$, green line in Fig. 6(a)] and, consequently, shear bands form at lower applied stresses and the glass yields also at lower stresses (see Supplemental Material, Fig. S7 and Table S2). While shear bands propagate at $42^{\circ}$ toward the center of the sample, the energy stored decreases because of the progressively adverse orientation of the elastic field [see profile in Fig. 6(b)], passes through an isoline where $W^{e f}=$ $W^{S T Z}\left(42^{\circ}\right)$, and finally enters in a region of low energies $\left[W^{e f}<W^{S T Z}\left(42^{\circ}\right)\right]$. In this region, shear band propagation at $42^{\circ}$ will be no longer energetically accessible; yet, the system continues to plastically deform through shear banding [Fig. 3(d)]. STZs must therefore be activated along a different direction, where higher stored energies provide an alternative, energetically more favorable path for shear band propagation. This hypothesis is corroborated by inspecting the values of $W^{e f}$ for $\theta=0^{\circ}$ [Fig. 6(c)]. In this case, the energy stored at the edges of the specimen is lower than the critical shear energy necessary for shear banding at $0^{\circ}\left[W^{e f}=W^{S T Z}\left(0^{\circ}\right)=\right.$ $0.0154 \mathrm{eV}$, green line in Fig. 6(c)]. Shear banding at $\theta=0^{\circ}$, however, becomes energetically possible moving toward the center of the specimen, where $W^{e f}>W^{S T Z}\left(0^{\circ}\right)$ [see profile in Fig. 6(d)], explaining the unusual shear band trajectory observed in the flash-annealed specimen [Fig. 3(d)].

\section{CONCLUSIONS}

Flash annealing provides a method capable to generate elastic stress fields in bulk metallic glasses without sacrificing structural integrity by forming shear bands. This enables us to directly explore the effect of residual stresses on shear banding. The direction of shear band propagation depends on the orientation of the superimposing stress field. We suggest this behavior to result from the altered propensity for STZ activation along the characteristic $42^{\circ}$ direction of the as-cast glass. Depending on their magnitude and orientation, residual stresses assist or oppose local atomic rearrangements, which proceed during shear transformations. Such a directionality can be taken into account by including the interaction of the superimposing stress field with all stress components resulting from STZ activation in the cooperative shear model to give the angular dependence of the tensorial shear stress. The contribution of the residual stresses is then reflected by the energy stored as shear in the STZ volume. When the energy stored along the path at $42^{\circ}$ is insufficient, the shear band will change its propagation direction. This is indeed observed in the flash-annealed glass, a phenomenon which renders them more malleable.

Although the generalized CSM proposed here provides a useful guideline for predicting the deviation of the shear band direction in the presence of a known superimposing stress field, the method is not yet able to capture all the observed phenomena; for example, the region where shear bands should propagate at $42^{\circ}$ predicted by Eq. (7) is larger than the area experimentally observed [compare Figs. 3(d) and 6(a)]. This discrepancy can be ascribed to the reference stress tensor used here to describe STZ activation $\left(\sigma^{S T Z}\right)$, which is necessarily incomplete due to experimental limitations. Because of the difficulties to identify a priori the location where a shear band will form, the present $\sigma^{S T Z}$ was obtained via ex situ measurements [8]. The effective energy derived using Eq. (7) is thus underestimated as it does not consider the contribution of the applied stress. Furthermore, we derived our results neglecting any contribution along the $z$ direction and assumed the material to be elastically homogeneous. A more realistic $\sigma^{S T Z}$ might be obtained in the future using in situ XRD strain tomography under loading, by which structural investigations can be extended to the missing direction and the effect of the applied stress and local variations of elastic moduli can be taken into account. The access to such an optimized $\sigma^{S T Z}$ would open up the possibility to effectively design advanced BMGs with predetermined shear band dynamics and hence tailored mechanical properties by stress engineering.

\section{ACKNOWLEDGMENTS}

K.K. and S.P. acknowledge financial support through the German Research Foundation (DFG) (Grants No. KO 5771/11 and No. PA 2275/6-1). Parts of this research were carried out at the light source PETRA III (beamline P02.1) at DESY, a member of the Helmholtz Association (HGF). Experimental support by K. Peukert, M. Ulbrich, D. Becker, U. Biscop, S. Donath, and N. Geißler is highly appreciated. We thank F. Dienerowitz for stimulating discussions.
[1] C. A. Schuh, T. C. Hufnagel, and U. Ramamurty, Acta Mater. 55, 4067 (2007).

[2] R. Maaß and J. F. Löffler, Adv. Funct. Mater. 25, 2353 (2015).

[3] V. Schmidt, H. Rösner, M. Peterlechner, G. Wilde, and P. M. Voyles, Phys. Rev. Lett. 115, 035501 (2015).

[4] R. Hubek, S. Hilke, F. A. Davani, M. Golkia, G. P. Shrivastav, S. V. Divinski, H. Rösner, J. Horbach, and G. Wilde, Front. Mater. 7144 (2020).

[5] D. Şopu, A. Stukowski, M. Stoica, and S. Scudino, Phys. Rev. Lett. 119, 195503 (2017).

[6] M. L. Falk and J. S. Langer, Phys. Rev. E 57, 7192 (1998).

[7] A. S. Argon, Acta Metall. 27, 47 (1979).

[8] S. Scudino and D. Şopu, Nano Lett. 16, 1221 (2018).
[9] K. Kosiba, D. Sopu, S. Scudino, L. Zhang, J. Bednarcik, and S. Pauly, Int. J. Plast. 119, 156 (2018).

[10] H. Wagner, D. Bedorf, S. Küchemann, M. Schwabe, B. Zhang, W. Arnold, and K. Samwer, Nat. Mater. 10, 439 (2011).

[11] P. Ross, S. Küchemann, P. M. Derlet, H. B. Yu, W. Arnold, P. Liaw, K. Samwer, and R. Maaß, Acta Mater. 138, 111 (2017).

[12] S. Pauly, S. Gorantla, G. Wang, U. Kühn, and J. Eckert, Nat. Mater. 9, 473 (2010).

[13] Y. Zhang, W. H. Wang, and A. L. Greer, Nat. Mater. 5, 857 (2006).

[14] S. Scudino, B. Jerliu, S. Pauly, K. B. Surreddi, U. Kühn, and J. Eckert, Scr. Mater. 65, 815 (2011). 
[15] S. Scudino, J. J. Bian, H. Shakur Shahabi, D. Şopu, J. Sort, J. Eckert, and G. Liu, Sci. Rep. 8, 9174 (2018).

[16] S. Scudino, B. Jerliu, K. B. Surreddi, U. Kühn, and J. Eckert, J. Alloys Compd. 509, S128 (2011).

[17] Q. P. Cao, J. W. Liu, K. J. Yang, F. Xu, Z. Q. Yao, A. Minkow, H. J. Fecht, J. Ivanisenko, L. Y. Chen, X. D. Wang, S. X. Qu, and J. Z. Jiang, Acta Mater. 58, 1276 (2010).

[18] K. Kosiba and S. Pauly, Sci. Rep. 7, 2151 (2017).

[19] H. E. Kissinger, Anal. Chem. 29, 1702 (1957).

[20] W. L. Johnson, G. Kaltenboeck, M. D. Demetriou, J. P. Schramm, X. Liu, K. Samwer, C. P. Kim, and D. C. Hofmann, Science 332, 828 (2011).

[21] K. Kosiba, S. Scudino, U. Kühn, A. L. Greer, J. Eckert, and S. Pauly, Acta Mater. 127, 416 (2017).

[22] See Supplemental Material at http://link.aps.org/supplemental/ 10.1103/PhysRevB.102.134113 for additional information regarding data acquisition and analysis, finite-element method simulations, mechanical properties, and shear band morphology.

[23] G. Ashiotis, A. Deschildre, Z. Nawaz, J. P. Wright, D. Karkoulis, F. E. Picca, and J. Kieffer, J. Appl. Crystallogr. 48, 510 (2015).

[24] S. Scudino, M. Stoica, I. Kaban, K. G. Prashanth, G. B. M. Vaughan, and J. Eckert, J. Alloys Compd. 639, 465 (2015).

[25] H. F. Poulsen, J. A. Wert, J. Neuefeind, V. Honkimaki, and M. Daymond, Nat. Mater. 4, 33 (2005).

[26] H. Shakur Shahabi, S. Scudino, U. Kühn, and J. Eckert, Mater. Des. 59, 241 (2014).

[27] S. Chen, L. Zhang, H. M. Fu, Z. K. Li, Z. W. Zhu, H. Li, H. W. Zhang, A. M. Wang, Y. D. Wang, and H. F. Zhang, Mater. Des. 160, 652 (2018).

[28] S. V. Ketov, Y. H. Sun, S. Nachum, Z. Lu, A. Checchi, A. R. Beraldin, H. Y. Bai, W. H. Wang, D. V. Louzguine-Luzgin,
M. A. Carpenter, and A. L. Greer, Nature (London) 524, 200 (2015).

[29] H. B. Yu, X. Shen, Z. Wang, L. Gu, W. H. Wang, and H. Y. Bai, Phys. Rev. Lett. 108, 015504 (2012).

[30] J. X. Zhao, F. F. Wu, R. T. Qu, S. X. Li, and Z. F. Zhang, Acta Mater. 58, 5420 (2010).

[31] X. D. Wang, R. T. Qu, S. J. Wu, Z. W. Zhu, H. F. Zhang, and Z. F. Zhang, Materialia 7, 100407 (2019).

[32] H. B. Yu, J. Hu, X. X. Xia, B. A. Sun, X. X. Li, W. H. Wang, and H. Y. Bai, Scr. Mater. 61, 640 (2009).

[33] M. H. Lee, K. S. Lee, J. Das, J. Thomas, U. Kühn, and J. Eckert, Scr. Mater. 62, 678 (2010).

[34] S. C. Lee, C. M. Lee, J. W. Yang, and J. C. Lee, Scr. Mater. 58, 591 (2008).

[35] S. H. Joo, D. H. Pi, A. D. H. Setyawan, H. Kato, M. Janecek, Y. C. Kim, S. Lee, and H. S. Kim, Sci. Rep. 5, 9660 (2015).

[36] Q. Wang, Y. Yang, H. Jiang, C. T. Liu, H. H. Ruan, and J. Lu, Sci. Rep. 4, 4757 (2014).

[37] S. Scudino and K. B. Surreddi, J. Alloys Compd. 708, 722 (2017).

[38] S. Scudino, J. Alloys Compd. 773, 883 (2019).

[39] J. Ketkaew, W. Chen, H. Wang, A. Datye, M. Fan, G. Pereira, U. D. Schwarz, Z. Liu, R. Yamada, W. Dmowski, M. D. Shattuck, C. S. O'Hern, T. Egami, E. Bouchbinder, and J. Schroers, Nat. Commun. 9, 3271 (2018).

[40] G. Kumar, P. Neibecker, Y. H. Liu, and J. Schroers, Nat. Commun. 4, 1536 (2013).

[41] W. L. Johnson and K. Samwer, Phys. Rev. Lett. 95, 195501 (2005).

[42] G. E. Dieter, Mechanical Metallurgy (McGraw-Hill, Singapore, 1988).

[43] S. T. Liu, Z. Wang, H. L. Peng, H. B. Yu, and W. H. Wang, Scr. Mater. 67, 9 (2012). 